\title{
Toll-like receptor 4 single-nucleotide polymorphisms Asp299Gly and Thr399Ile in ovarian cancers
}

\author{
AN-CONG WANG ${ }^{1,2}$, FENG-XIA WU ${ }^{3}$, YONG-SHENG GAO ${ }^{4}$ and XIU-GUI SHENG ${ }^{2}$ \\ ${ }^{1}$ Department of Reproductive Medicine, Linyi People's Hospital, Linyi, Shandong 276003; \\ ${ }^{2}$ Department of Gynecologic Oncology, Shandong Cancer Hospital and Institute, Jinan, Shandong 250117; \\ ${ }^{3}$ Department of Anatomy, Shandong University, Jinan, Shandong 250012; ${ }^{4}$ Department of Pathology, \\ Shandong Cancer Hospital and Institute, Jinan, Shandong 250117, P.R. China
}

Received September 24,2013; Accepted April 1, 2014

DOI: $10.3892 / 01.2014 .2113$

\begin{abstract}
Toll-like receptor (TLR4) 4 is present in numerous cell types and serves as the first point of defense in the innate immune system. Single-nucleotide polymorphisms (SNPs) are present in a number TLR genes and have been associated with various infection and inflammation disorders. Asp299Gly and Thr399Ile, TLR4 SNPs, are associated with tumor progression. In the present study, cases of ovarian cancer were analyzed with regards to Asp299Gly and Thr399Ile of the TLR4 gene. Genotype analysis was performed using DNA from tissue samples from stage I-IV patients with ovarian cancer. DNA from tissue samples was extracted and analyzed by a pyrosequencing method following multiplex polymerase chain reaction. The genotypes of these SNPs were analyzed in the present study in a population of 105 patients, with different types of ovarian cancer, between 2004 and 2012. The allele frequencies for TLR4 Asp299Gly identified in this population were $1.00(\mathrm{~A})$ and $0.00(\mathrm{G})$; for TLR4 Thr399Ile the allele frequencies were; $1.00(\mathrm{C})$ and $0.00(\mathrm{~T})$. For TLR4 Asp299Gly the observed genotype frequency was 1.00 (AA), 0.00 (AG) and 0.0 (GG). In TLR4 Thr399Ile the observed genotype frequencies were 1.00 (CC), 0.00 (CT) and 0.00 (TT). TLR4 Asp299Gly and Thr399Ile alleles were not detected in the patients. These results indicated that the TLR4 299Gly and 399Ile alleles were exhibited at a lower frequency in the ovarian cancer patients that were examined.
\end{abstract}

Correspondence to: Professor Xiu-Gui Sheng, Department of Gynecologic Oncology, Shandong Cancer Hospital and Institute, 440 Jiyan Road, Jinan, Shandong 250117, P.R. China

E-mail: shengxgjnsd@163.com

Key words: toll-like receptor 4, single-nucleotide polymorphism, ovarian cancer

\section{Introduction}

Chronic infection and inflammation are important epigenetic factors contributing to tumorigenensis and tumor progession (1). Toll-like receptors (TLRs) are important pattern recognition receptors expressed by immune cells. Toll-like receptor 4 (TLR4) expression has been investigated in tumor cells or cell lines, including gastric carcinoma, extranodal marginal zone B-cell lymphomas, pituitary epithelial tumor cell lines, hepatocellular carcinoma cells, colon cancer cells and human prostate epithelial PC3 cells. Although TLR4 is expressed in numerous non-immune cells and tumor cells, the functional association of TLR4 with tumor progression requires further elucidation. The TLR4 single-nucleotide polymorphisms (SNPs), Asp299 and Thr399, have been reported to be involved in inflammation and cancer $(2,3)$.

It was hypothesized that the presence of TLR4 variants may lead to the development of ovarian cancer. In the present study, the relevance of TLR4 SNPs, Asp299Gly (rs4986790) and Thr399Ile (rs4986791) are investigated in 105 ovarian cancer patients retrospectively with an extended follow-up and complete representative adjuvant therapy (chemotherapy with or without surgical treatment).

\section{Patients and methods}

Patients and tissue samples. Tissue specimens of 105 ovarian cancer patients (mean age, $54 \pm 11.7$ years) were collected by the Department of Pathology, Shandong Cancer Hospital and Institute (Jinan, China). All patients were diagnosed and treated at the Department of Gynecologic Oncology, Shandong Cancer Hospital and Institute between 2004 and 2012. Treatment strategies were determined based on consensus recommendations from gynecological oncologists, which were based on guidelines for ovarian cancer treatment at the time (4). All patients provided written informed consent for the use of their tissues and participation in the study. The tissue samples were obtained during diagnostic or therapeutic surgery. Overall, 75 (71\%) patients received paclitaxel + carboplatin chemotherapy prior or subsequent to surgery. A total of $19(18 \%)$ patients received other chemotherapy prior or following surgery, and $11(11 \%)$ patients received only surgery. Regular follow-up procedures 


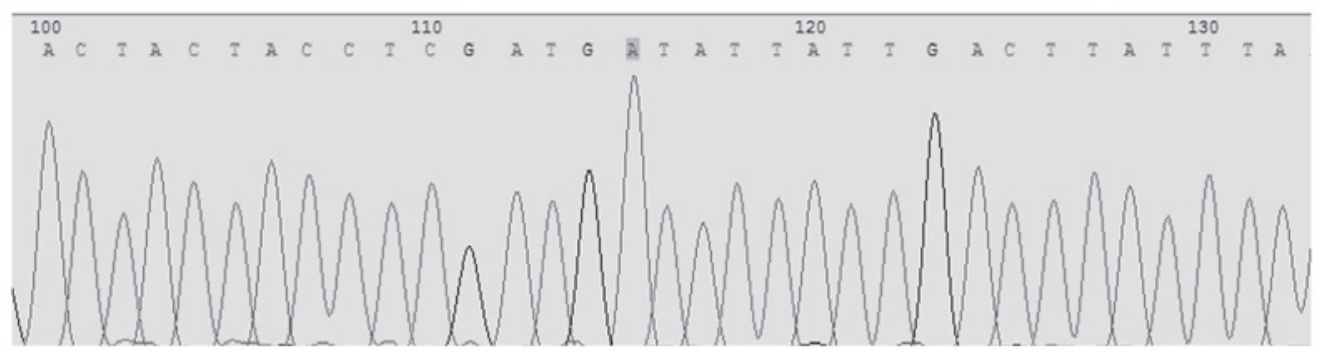

Figure 1. Sequence analysis of TLR4 Asp299Gly polymorphisms in ovarian cancer patients. The results of genoytype sequence CGATG $\underline{\text { ATATTA. }}$

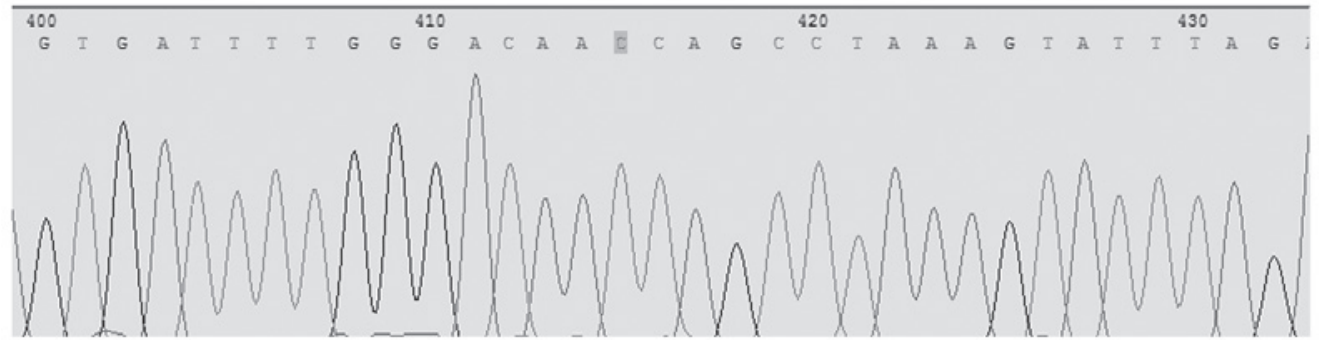

Figure 2. Sequence analysis of TLR4 Thr399Ile polymorphisms in ovarian cancer patients. The results of genoytype sequence GACAACCAGCC.

were performed and the median follow-up in patients who had survived until the time of analysis was $26 \pm 17.6$ months (range, 1-96 months).

Sequence analysis of TLR4. As described in a previous study (5), DNA samples were extracted from $10-\mu \mathrm{m}$ sections of formalin-fixed, paraffin-embedded tumor tissue. The germline mutations, TLR4 Asp299Gly (rs4986790) and Thr399Ile (rs4986791) were analyzed in all patients using pyrosequencing. For TLR4 Asp299Gly (rs4986790) and Thr399Ile (rs4986791), pyrosequencing was performed with the forward primer, 5'-TCTGGCTGGTTTAGAAGTCCA-3'; and the reverse primer, 5'-AATTGCCAGCCATTTTCAAG-3'; resulting in a 698-bp fragment. Following denaturation at $95^{\circ} \mathrm{C}, 35$ cycles of DNA amplification were performed using Taq DNA Polymerase 2X Master Mix Red (Ampliqon-Biomol, Hamburg, Germany) at $95^{\circ} \mathrm{C}$ for $30 \mathrm{sec}, 60^{\circ} \mathrm{C}$ for $30 \mathrm{sec}$ and $72^{\circ} \mathrm{C}$ for $60 \mathrm{sec}$, with a final extension for $5 \mathrm{~min}$ at $72^{\circ} \mathrm{C}$. Once the amplification was confirmed, the polymerase chain reaction product was digested for $1 \mathrm{~h}$ at $37^{\circ} \mathrm{C}$ with the restriction enzyme (Invitrogen Life Technologies, Carlsbad, CA, USA), Ncol (6). The SNP assays were purchased from Applied Biosystems, Inc., (ABI; Carlsbad, CA, USA) and performed on the ABI StepOnePlus ${ }^{\mathrm{TM}}$ system. Data were analyzed using ABI StepOne ${ }^{\mathrm{TM}}$ Software.

\section{Results}

Pyrosequencing was conducted for the simultaneous detection of Asp299Gly (rs4986790) and Thr399Ile (rs4986791) in the TLR4 gene. The allele frequencies for TLR4 Asp299Gly identified in this population were $1.00(\mathrm{~A})$ and $0.00(\mathrm{G})$; for TLR4 Thr399Ile the allele frequencies were, 1.00 (C) and 0.00 (T). For TLR4 Asp299Gly the observed genotype frequency was $1.00(\mathrm{AA}), 0.00(\mathrm{AG})$ and $0.0(\mathrm{GG})$. In TLR4
Thr399Ile the observed genotype frequencies were 1.00 (CC), 0.00 (CT) and 0.00 (TT; Figs. 1 and 2).

\section{Discussion}

Chronic inflammation is significant in the progression of various human cancers. Previous studies have revealed that inflammation-induced TLRs are involved in tumorigenesis (7-11). Additionally, certain studies have shown that the activation of TLR4 signaling may correlate with tumor progression (12). Thus, TLRs may be candidates as independent prognostic cancer markers (13).

The response to TLR ligands may be impaired by SNPs that are present in TLR genes, resulting in a modified susceptibility to the outcome of infectious or inflammatory diseases. Furthermore, the TLR genes have been shown to be polymorphic (6).

Currently, two SNPs within the human TLR4 gene have been focused on. The first is an A-G substitution at the 896-bp region, which results in an aspartic acid to glycine replacement at the 299 position of the amino acid sequence (Asp299Gly), and the other is a C-T substitution at the 1,196-bp region, which results in a threonine to isoleucine exchange at position 399 in the amino acid sequence (Thr399Ile) (2).

In breast cancer, data shows that patients who express the TLR4 loss of function allele experience a relapse more rapidly, following radiotherapy and chemotherapy, when compared with those patients who express the normal allele (14). For marginal zone B cells, TLR4 is the major receptor for lipopolysaccharide, and the rare TLR4 Asp299Gly allele attenuates the receptor signaling and decreases the inflammatory response. Data has shown that heterozygous genotypes are expressed significantly less frequently in patients with gastric mucosa-associated lymphoid tissue (MALT) lymphoma compared with that of 
Helicobacter pylori-infected control subjects. The TLR4 Asp299Gly genotype acts as a protective factor during the development of gastric MALT lymphoma in Caucasians (15).

Notably, there is an opposite conclusion for the TLR4 Asp299Gly/Thr399Ile polymorphism that is associated with gastric cancer. A study by Kato et al (16) reported no association between the TLR4 Asp299Gly polymorphism and gastric pre-cancerous lesions. However, de la Trejo et al (17) reported that the Asp299Gly polymorphism in TLR4 was significantly associated with duodenal ulcers and that there was a trend for an association with gastric cancer, with Asp299Gly polymorphism values similar in patients with or without the H. pylori infection. The Thr399Ile polymorphism in TLR4 was also identified as a genetic risk factor for gastritis and pre-cancerous lesions in a northern Indian population (18). Santini et al (19) demonstrated that the TLR4 Thr399Ile polymorphism is linked with an increased susceptibility to gastric cancer. However, other data indicates that TLR4 Asp299Gly and Thr399Ile are extremely rare in the Japanese population and, therefore, they may not be significant factors in establishing the outcome of $H$. pylori-infected Japanese patients (20). Guo et al (21) demonstrated that the polymorphism of cluster of differentiation, but not the TLR4 Asp299Gly mutation, was associated with a presence of colorectal cancer in Chinese patients. Zhang et al (22) indicated that using additional genetic models for rs4986790 and rs4986791 complicates analysis. Their meta-analysis indicated that the two SNPs (rs4986790 and rs4986791) in TLR4 were associated with an increased cancer risk, however, one SNP in TLR4 (rs1927911) was associated with a decreased cancer risk. Thus, the frequency of different polymorphisms has been shown to vary significantly across the different ethnic populations worldwide.

In conclusion, the TLR4 Asp299Gly and Thr399Ile alleles were not detected in the ovarian cancer patients in the present study. The results indicate that the TLR4 299Gly and 399Ile alleles have a markedly reduced frequency in northern Chinese ovarian cancer patients compared with those presented in the study by Zhang et al (21). Although detailed mechanisms and regulation of the TLR4 functions in tumor pathogenesis remain to be elucidated, TLR4 may be a promising target for the development of anticancer agents in the future.

\section{References}

1. Balkwill F and Coussens LM: Cancer: an inflammatory link Nature 431: 405-406, 2004.

2. Arbour NC, Lorenz E, Schutte BC, et al: TLR4 mutations are associated with endotoxin hyporesponsiveness in humans. Nat Genet 25: 187-191, 2000.

3. Lorenz E, Patel DD, Hartung T and Schwartz DA: Toll-like receptor 4 (TLR4)-deficient murine macrophage cell line as an in vitro assay system to show TLR4-independent signaling of Bacteroides fragilis lipopolysaccharide. Infect Immun 70 4892-4896, 2002.
4. Poveda Velasco A, Casado Herráez A, Cervantes Ruipérez A, et al: Treatment guidelines in ovarian cancer. Clin Transl Oncol 9: 308-316, 2007.

5. Lehnerdt GF, Franz P, Zaqoul A, et al: Overall and relapse-free survival in oropharyngeal and hypopharyngeal squamous cell carcinoma are associated with genotypes of T393C polymorphism of the GNAS1 gene. Clin Cancer Res 14: 1753-1758, 2008.

6. Montes AH, Asensi V, Alvarez V, et al: The Toll-like receptor 4 (Asp299Gly) polymorphism is a risk factor for Gram-negative and haematogenous osteomyelitis. Clin Exp Immunol 143: 404-413, 2006.

7. Fukata M, Chen A, Vamadevan AS, et al: Toll-like receptor-4 promotes the development of colitis-associated colorectal tumors. Gastroenterology 133: 1869-1881, 2007.

8. Lee SJ and Lim KT: UDN glycoprotein regulates activities of manganese-superoxide dismutase, activator protein-1, and nuclear factor-kappaB stimulated by reactive oxygen radicals in lipopolysaccharide-stimulated HCT-116 cells. Cancer Lett 254: 274-287, 2007.

9. Wang JH, Manning BJ, Wu QD, Blankson S, Bouchier-Hayes D and Redmond HP: Endotoxin/lipopolysaccharide activates NF-kappa B and enhances tumor cell adhesion and invasion through a beta 1 integrin-dependent mechanism. J Immunol 170: 795-804, 2003.

10. Cianchi F, Cortesini C, Fantappiè O, et al: Cyclooxygenase-2 activation mediates the proangiogenic effect of nitric oxide in colorectal cancer. Clin Cancer Res 10: 2694-2704, 2004.

11. Molteni M, Marabella D, Orlandi C and Rossetti C: Melanoma cell lines are responsive in vitro to lipopolysaccharide and express TLR-4. Cancer Lett 235: 75-83, 2006.

12. Huang B, Zhao J, Li H, et al: Toll-like receptors on tumor cells facilitate evasion of immune surveillance. Cancer Res 65: 5009-5014, 2005.

13. Castro FA, Försti A, Buch S, et al: TLR-3 polymorphism is an independent prognostic marker for stage II colorectal cancer. Eur J Cancer 47: 1203-1210, 2011.

14. Apetoh L, Ghiringhelli F, Tesniere A, et al: Toll-like receptor 4-dependent contribution of the immune system to anticancer chemotherapy and radiotherapy. Nat Med 13: 1050-1059, 2007.

15. Hellmig S, Fischbach W, Goebeler-Kolve ME, Fölsch UR, Hampe $\mathrm{J}$ and Schreiber S: Association study of a functional Toll-like receptor 4 polymorphism with susceptibility to gastric mucosa-associated lymphoid tissue lymphoma. Leuk Lymphoma 46: 869-872, 2005.

16. Kato I, Canzian F, Plummer M, et al: Polymorphisms in genes related to bacterial lipopolysaccharide/peptidoglycan signaling and gastric precancerous lesions in a population at high risk for gastric cancer. Dig Dis Sci 52: 254-261, 2007.

17. Trejo-de la OA, Torres J, Pérez-Rodríguez M, et al: TLR4 single-nucleotide polymorphisms alter mucosal cytokine and chemokine patterns in Mexican patients with Helicobacter pylori-associated gastroduodenal diseases. Clin Immunol 129: 333-340, 2008.

18. Achyut BR, Ghoshal UC, Moorchung N and Mittal B: Association of Toll-like receptor-4 (Asp299Gly and Thr399Ileu) gene polymorphisms with gastritis and precancerous lesions. Hum Immunol 68: 901-907, 2007.

19. Santini D, Angeletti S, Ruzzo A, et al: Toll-like receptor 4 Asp299Gly and Thr399Ile polymorphisms in gastric cancer of intestinal and diffuse histotypes. Clin Exp Immunol 154: 360-364, 2008.

20. Tahara T, Arisawa T, Shibata T, Hirata I and Nakano H: Absence of common polymorphisms of toll like receptor 4 (TLR4): Asp299Gly, Thr399Ile in patients with gastroduodenal diseases in Japan. J Clin Biochem Nutr 40: 62-65, 2007.

21. Guo Q, Zhu J and Xia B: Polymorphism of CD14 gene but not the mutation of TLR4 gene is associated with colorectal cancer in Chinese patients. J Gastroenterol Hepatol 21: 92-77, 2006.

22. Zhang K, Zhou B, Wang Y, Rao L and Zhang L: The TLR4 gene polymorphisms and susceptibility to cancer: a systematic review and meta-analysis. Eur J Cancer 49: 946-954, 2013. 